\title{
LES-RANS of installed ultra-high bypass-ratio coaxial jet aeroacoustics with a finite span wing-flap geometry and flight stream - Part 1: round nozzle
}

\author{
James C. Tyacke* Zhong-Nan Wang ${ }^{\dagger}$ \\ and Paul G. Tucker ${ }^{\ddagger}$ \\ Department of Engineering, University of Cambridge, Cambridge, UK
}

\begin{abstract}
The jet noise produced by aeroengines is a critical topic in engine design. Large-Eddy Simulation (LES) and hybrid LES-Reynolds-Averaged Navier-Stokes (LES-RANS), provides a method to increase understanding of influences on the noise produced. Installed jet noise modelling has received less attention than isolated jet noise yet is becoming more important for design. Here, using LES-RANS, a coaxial nozzle with an ultra-high bypassratio of 15 is studied with and without a wing-flap geometry. The bypass ratio leads the nozzle to become extremely close to the wing-flap geometry introducing strong installation effects. Two different flap deflections of 8 and 14 degrees are contrasted with an isolated round nozzle. A flight stream is applied and an FWH surface placement procedure for installed jets is proposed. The installed cases generate more directional noise at mid-low frequencies as the presence of the flap trailing edge produces a strong dipole source. Second order space-time correlations reveal length and time scales int he flow. Fourth order spacetime correlations indicate increasing magnitudes of the dominant noise source components with in increasing flap angle and may lead to improved acoustics models.
\end{abstract}

*Senior Research Associate, Department of Engineering, University of Cambridge, Cambridge, CB3 0DY, member AIAA.

${ }^{\dagger}$ Research Associate, Department of Engineering, University of Cambridge, Cambridge, CB3 0DY, member AIAA

$\ddagger$ Professor, Department of Engineering, University of Cambridge, Cambridge, CB3 0DY, Associate fellow AIAA. 


\section{Nomenclature}

$\begin{array}{ll}d_{\text {wall }} & =\text { Wall distance }(\mathrm{m}) \\ f & =\text { frequency }(\mathrm{Hz}) \\ F_{i j} & =\text { Edge flux } \\ D & =\text { bypass nozzle diameter }(\mathrm{m}) \\ \bar{L} & =\text { Linear modelled stress } \\ \overline{N L} & =\text { Nonlinear modelled stress } \\ P_{0} & =\text { total pressure }(\mathrm{Pa}) \\ P_{a} & =\text { ambient pressure }(\mathrm{Pa}) \\ R & =\text { Radius }(\mathrm{m}) \\ S t & =\text { Strouhal number } \\ T_{0} & =\text { total temperature }(\mathrm{K}) \\ T_{a} & =\text { ambient temperature }(\mathrm{K}) \\ u & =\text { Cartesian velocity component }(\mathrm{m} / \mathrm{s}) \\ u_{B P} & =\text { bypass stream axial velocity }(\mathrm{m} / \mathrm{s}) \\ u_{c} & =\text { core stream axial velocity }(\mathrm{m} / \mathrm{s}) \\ x, y, z & =\text { Cartesian coordinates }(\mathrm{m}) \\ \delta & =\text { Boundary layer thickness }(\mathrm{m}) \\ \tau_{i j} & =\text { Modelled stress } \\ \varepsilon & =\text { Smoothing parameter } \\ \mathrm{F} 14 & =14 \text { deg. flap angle } \\ \mathrm{F} 8 & =8 \text { deg. flap angle } \\ \mathrm{FS} & =\text { flight stream } \\ \mathrm{FO} & =\text { Flyover } \\ \mathrm{SL} & =\text { Sideline } \\ \mathrm{OASPL} & =\text { overall sound pressure level }(\mathrm{dB}) \\ \phi & =\text { Polar angle }(\text { degrees }) \\ \theta & =\text { Azimuthal angle }(\text { degrees }) \\ & \end{array}$

\section{Introduction}

The noise produced by aeroengines is a critical topic in engine design. The increase in air travel and tightening emissions targets require both airframe and aeroengine designers to accurately predict performance and pollutant and noise emissions. The Advisory Council for Aeronautical Research in Europe (ACARE) has set the target of a reduction in perceived noise emission of $50 \%$ (to those of the year 2000) by the year 2020. To enable such challenging targets to be met, the importance of the many influences on jet noise must be understood. Engine-airframe design is becoming more closely coupled, hence, installation effects are the main focus of this paper, where an isolated round nozzle is contrasted with an installed nozzle with two flap deflections.

The trend towards higher bypass ratio engines and closer integration with the airframe constantly changes the design space. This makes the use of simple correlations less attractive if they are unable to capture the numerous effects on noise adequately. In fact Viswanathan et al. ${ }^{1}$ suggest there is a strong need to update existing methods. Experimental facilities are expensive and it can be difficult to obtain certain data, for example to include flight stream effects. The multiple unsteady interactions for complex geometry jets make RANS unsuitable do to separation, recirculation, re-laminarisation, mixing and strong streamline curvature among others. Set in this backdrop, eddy resolving methods such as LES and hybrid LES-RANS seem attractive.

The Ffowcs-Williams Hawkings (FWH) approach has been used with much success in conjunction with LES for the prediction of jet noise. ${ }^{2}$ The use of a permeable FWH surface surrounding all noise sources avoids the expensive computation of volume source terms throughout the domain. For axi-symmetric domains, use 
of multiple FWH surfaces ensures the best surface placement ${ }^{2,3}$ (near the jet but far enough away from hydrodynamic effects). Nelson et al. ${ }^{4}$ also provide some guidance on grid generation and FWH surface placement for an axi-symmetric single stream jet. A summary of our recent practice and validation is provided by Tyacke et al. ${ }^{5}$ For complex geometries the optimum FWH surface placement is not clear $a$ priori. Paliath and Premasuthan ${ }^{6}$ use a simplified surface that encompasses the entire geometry a nearly uniform distance from the geometry. Another method is to base the FWH surface on an iso-surface of mean turbulence kinetic energy. ${ }^{7}$ The introduction of a flight stream compounds static jets issues, strongly convecting vortices downstream making FWH surface closure problematic. Rahier et al. ${ }^{8}$ use approximated quadrupole terms to effectively close the FWH surface reducing FWH placement and closure sensitivity. Pérez et al. ${ }^{9}$ filter the acoustic component from the near field which may also be useful. Installed nozzle with a flight stream will generate low frequencies downstream and hence requires a long FWH surface. This may adequately capture low polar angle noise without any special end disc treatment if other hydrodynamic regions are avoided near the control surfaces and jet plume.

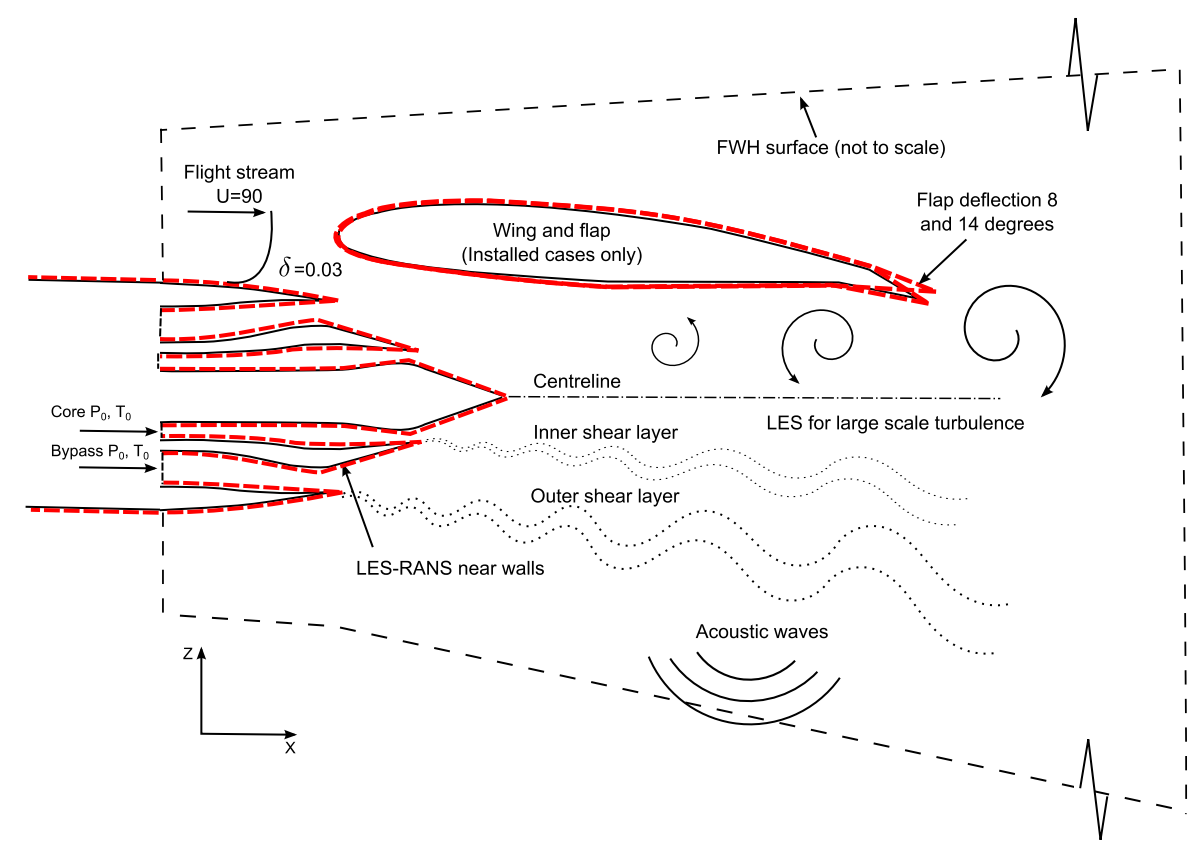

Figure 1. Case setup schematic indicating boundary conditions and geometry features in the $y=0$-plane.

Figure 1 indicates the main features of the geometry, modelling, flow and boundary conditions used.

The paper is structured in the following way. The numerical methods including the solver, turbulence modelling and acoustic processing are discussed. The the mesh and case setup are described. The results including flow, space-time correlations and acoustics are then presented and conclusions drawn.

\section{Numerical Methods}

\section{A. Solver}

An unstructured finite volume, edge-based compressible solver is used. Second order backward differencing is used for time and a second order kinetic energy preserving discretisation ${ }^{10}$ is used in space with numerical smoothing adapted for jet simulations. The KEP cell face flux with additional numerical smoothing is given by equation (1).

$$
F_{i j}=\frac{1}{2}\left(\rho_{i}+\rho_{j}\right) \times \frac{1}{2}\left(u_{i}+u_{j}\right) \times \frac{1}{2}\left(\phi_{i}+\phi_{j}\right)-\frac{1}{2} \varepsilon|A|\left[\nabla_{i}^{2} \phi-\nabla_{j}^{2} \phi\right]
$$

The parameter $\varepsilon$ is used to minimise numerical dissipation in the central jet flow and FWH region and is increased along with mesh expansion to form sponge zones near the farfield boundaries to prevent wave reflections. This is shown in Fig. 2. 


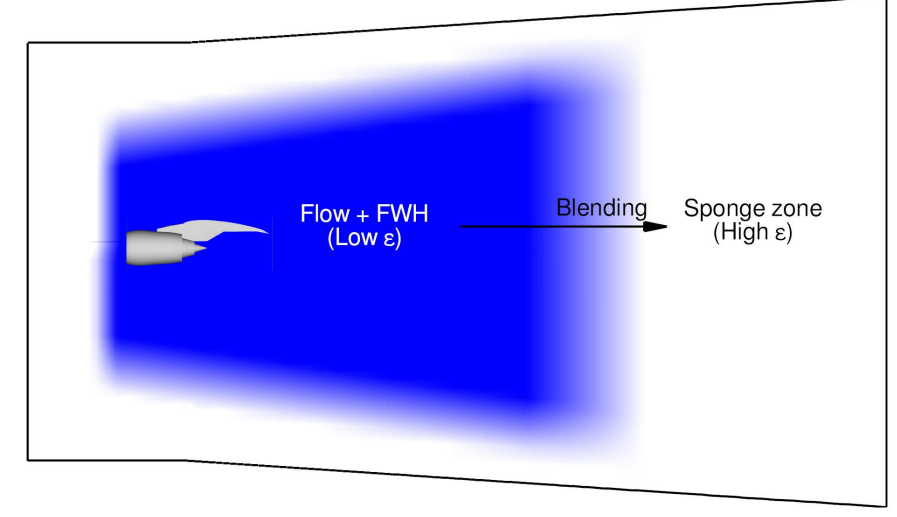

Figure 2. Numerical smoothing applied in the computational domain (not to scale).

\section{B. Turbulence modelling}

To alleviate near wall grid requirements a hybrid LES-RANS model is used. LES-RANS has been used with success for jets and other complex flows. ${ }^{5,11-14}$ Near walls the Spalart-Allmaras RANS model ${ }^{15}$ is used to model the inner boundary layer. Outside of the RANS layer where larger scales dominate, a mixed nonlinear alpha LES model is used ${ }^{16}$ to resolve complex unsteady flow features. The true wall distance $d_{\text {wall }}$ is retained in the RANS region, then smoothly returns the wall distance to zero for the LES region. The Smagorinsky SGS model ${ }^{17}$ is used to provide dissipation with a constant of $C_{s}=0.1$ and the mixed alpha model is used to add non-linear terms, modelling regions of anisotropy. Upstream of the potential core, the SGS filter is defined as $\Delta_{S G S}=\left(\Delta_{x} \Delta_{y} \Delta_{z}\right)^{(1 / 3)}$ to appropriately capture shear layer development. After the end of the potential core $(x / D \approx 10), \Delta_{S G S}=\max \left(\Delta_{\max },\left(\Delta_{x} \Delta_{y} \Delta_{z}\right)^{(1 / 3)}\right)$ in order to suitably model the more isotropic turbulence downstream. To provide a smooth transition from the RANS to LES, a function is used to modify the wall distance and blend the two models. The overall model is described by Equations $2-4$.

$$
\begin{gathered}
\tau_{i j}^{\text {model }}=\bar{L}+\overline{N L} \\
\bar{L}=\frac{\tau_{k k} \delta_{i j}}{3}+2\left[f \cdot \mu_{S G S}+(1-f) \cdot \mu_{R A N S}\right] S_{i j} \\
\overline{N L}=\rho C_{\alpha} \Delta_{S G S}^{2} f\left(\frac{\partial u_{i}}{\partial x_{l}} \frac{\partial u_{l}}{\partial u_{j}}+\frac{\partial u_{i}}{\partial x_{l}} \frac{\partial u_{j}}{\partial u_{l}}+\frac{\partial u_{l}}{\partial x_{i}} \frac{\partial u_{l}}{\partial u_{j}}\right)
\end{gathered}
$$

$S_{i j}=0.5\left(\partial u_{i} / \partial x_{j}+\partial u_{j} / \partial x_{i}\right)$ is the strain rate tensor and the blending function is given as

$$
f=\min \left[\max \left(\frac{d_{\text {wall }}-(1-\beta) d_{R A N S}}{\beta d_{R A N S}}, 0\right), 1\right]
$$

where $d_{R A N S}$ is the RANS layer thickness and $\beta$ defines the size of the RANS to LES transition zone as a fraction of $d_{R A N S}$. To account for different fluid stream velocities, $d_{R A N S}$ is set locally to cover the inner layer.

\section{Acoustic processing}

A permeable FWH surface is used to record time series of the primitive variables for later processing using an FWH solver. The use of multiple FWH surfaces has proven to be useful in ensuring only acoustic signals are being recorded at the surface and not vortical/hydrodynamic signals. The ideal placement of FWH surfaces is challenging a priori, hence the use of multiple surfaces allows data to be recorded and assessed later. Defining an FWH surface for an installed jet is challenging in itself. The addition of a flight stream however introduces strong wing-flap interaction with jet plume deflection and shed vortices that travel far 
downstream. It was decided to use conservative geometry and flow based criteria to determine the surfaces used. Three criteria were defined based on wall distance, turbulence intensity and non-dimensional vorticity. These are $d_{\text {wall }}>0.25 D$, Ti $<0.25 \%$ and $|\Omega| D / u_{B P}<0.05$ respectively. The two flow criterion are indicated in Fig. 3(a). The outermost of any of these surfaces was chosen to define a complex 3D surface extending throughout the domain in the upstream and downstream directions. This was then offset in the surface normal direction several times as shown in Fig. 3(b). Multiple closing discs with different axial and radial limits were also created from these as shown in the previous figures. Hence data is recorded for numerous scenarios and further processing. The innermost surfaces are shown in more detail in Figs. 3(c)-3(d). The surrounded nozzle, wing, tip vortices and eventual jet plume return to roundness is visible. Results presented use upstream closing surfaces to capture installation noise at high polar angles. The downstream end is left open but a sufficiently long surface ensures sound sources are captured at low polar angles.

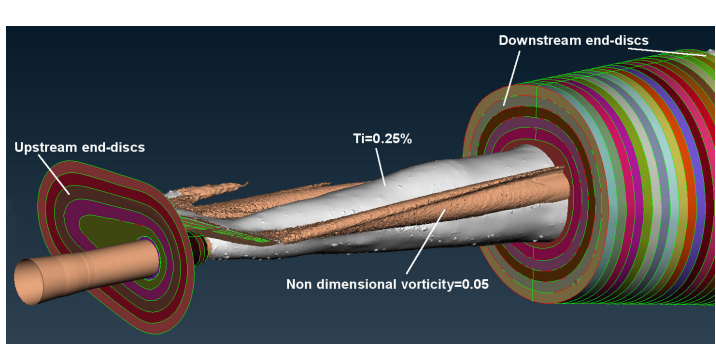

(a)

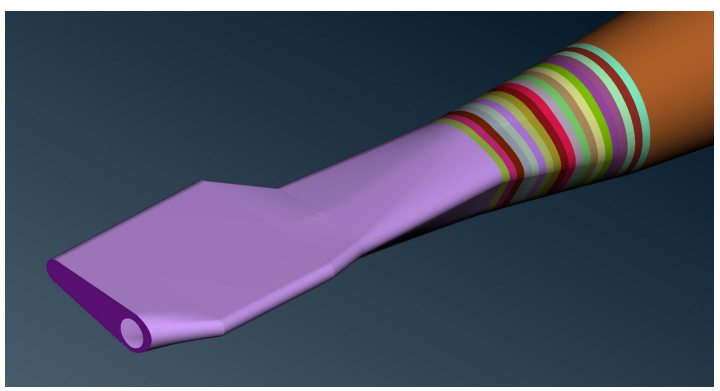

(c)

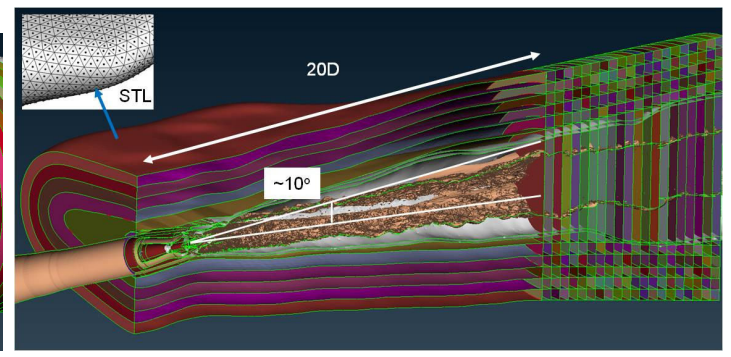

(b)

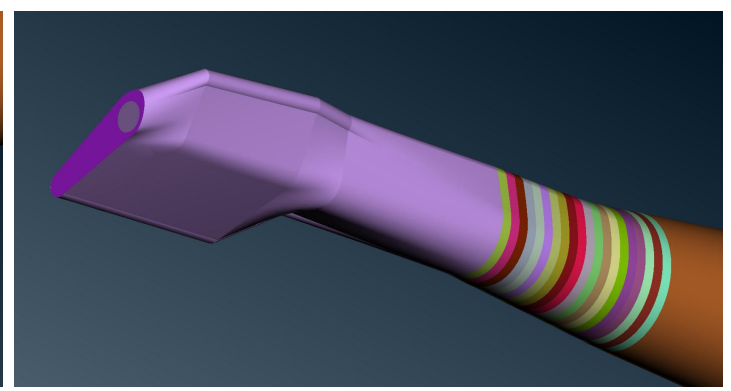

(d)

Figure 3. FWH surface placement.

The convective formulation of the FWH equation of ${ }^{18}$ is used to project the near field source to the far-field.

\section{Mesh}

For all simulations a hybrid structured-unstructured mesh is used. A structured hexahedral mesh is utilised in the jet plume region. This is less dissipative and fills volumes efficiently. Outside of the jet plume, unstructured tetrahedral mesh is used. This can be seen in Fig. 4(a). This allows the complex wing-flap geometry to be embedded easily and provides more mesh consistency between simulations. At the interface the ratio of $\Delta x$ and $R \Delta \theta$ is kept below $2 .{ }^{19}$ As the number of azimuthal and radial nodes are fixed, the interface relies on the axial distribution. In all meshes the axial expansion ratio is kept between $0.5-1.0 \%$. Near the nozzle, a 2D $(x-R)$ quad-dominant mesh used to prevent fine mesh radiating outwards and reconciles the interface radial location which lies within the nozzle for small mesh spacings. This mesh is extruded circumferentially with a uniform spacing giving a total of $160 \Delta \theta$. As shown in Fig. 4(b), for installed cases, the jet plume is deformed to follow the shear layer trajectories under the wing-flap. This is done following a short run on a preliminary mesh. The wing and flap geometries are surface-meshed using $2 \mathrm{D}$ structured and quad-dominant unstructured mesh. Layers are then extruded in the wall normal direction. The quad dominant surface mesh gives hexahedral prism cells predominantly aligned with the mean flow in boundary layer regions. To avoid jumps in mesh resolution at interfaces between the prism layers and the outer tetrahedral mesh, the surface mesh resolution is chosen based on the growth rate and number of 


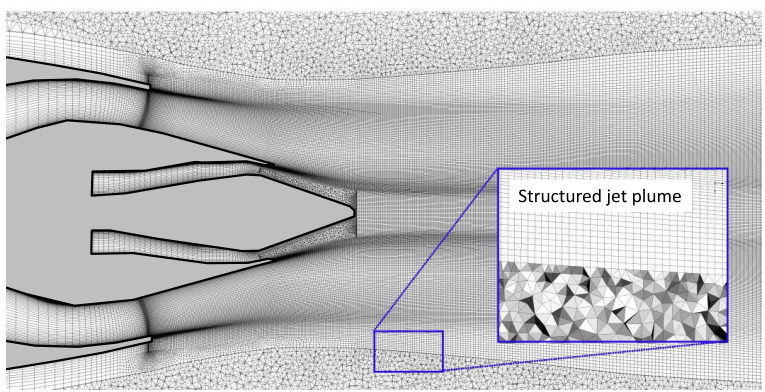

(a) Round nozzle

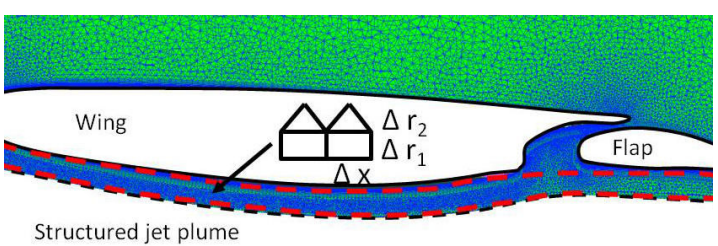

(c) Installed mesh interface $x-R$

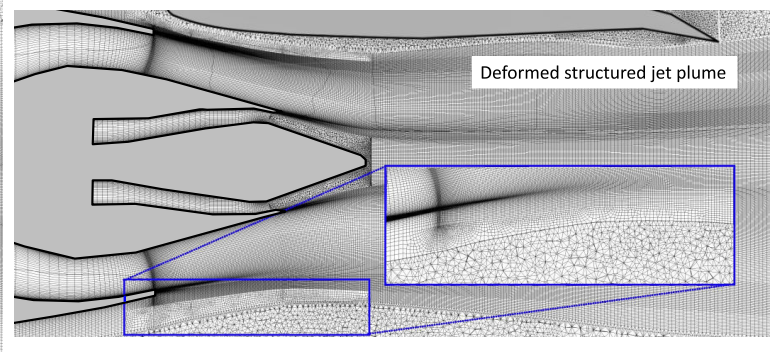

(b) Installed round nozzle

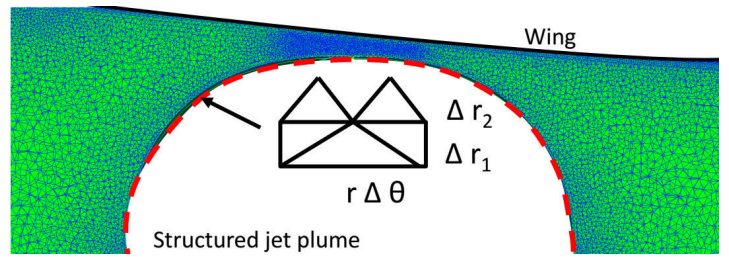

(d) Installed mesh interface $R-\theta$

Figure 4. Hybrid structured-unstructured mesh for (a), an isolated nozzle with the inset showing the structured-unstructured interface and (b), an initial installed round coaxial nozzle with an inset showing regions of different axial resolution,(c), structured-unstructured interface in the $x-R$ plane,(d), structuredunstructured interface in the $R-\theta$ plane.

layers so that the final wall normal spacing matches the surface mesh spacing within a factor of two giving a reasonably isotropic cell aspect ratio. An additional restriction is to match the outer mesh spacing of the structured jet plume mesh with the surface mesh requirements of the wing-flap prism layers. The mesh near the nozzle is of a similar scale in the $x$ and $R$ directions. A gap of approximately 8-10 average mesh spacings was left between the deformed structured mesh and the outer wig-flap layers to allow a smooth transition. This is indicated in in Fig. 4(c) by the dashed region. As the tetrahedral cells are isotropic, to match the $R \Delta \theta$ spacing, the number of $\theta$ points was locally doubled in the upper plume interface so that the $R \Delta \theta \approx \Delta R \approx \Delta x$. This is indicated in Fig. 4(d) by the dashed line. Based on expected frequencies at different axial and radial locations, a nest of cylindrical volumes were used to limit maximum mesh spacing and expansion ratio of $1.05-1.06$ up to the last FWH surfaces where the expansion ratio was then relaxed to 1.2 for the sponge region. To total cell counts for the isolated and installed cases are approximately 60 million and 130 million respectively.

\section{Case setup}

Table 1. Jet nozzle boundary conditions.

\begin{tabular}{lcc}
\hline & Bypass & Core \\
\hline$P_{0} / P_{a}(\mathrm{~Pa})$ & 1.342 & 1.222 \\
$T_{0} / T_{a}(\mathrm{~K})$ & 1.128 & 2.652 \\
$\dot{m}\left(\mathrm{Kgs}^{-} 1\right)$ & 6.373 & 0.414 \\
\hline
\end{tabular}

Table 1 provides a summary of the nozzle conditions as indicated in Fig. 1. The boundary conditions for the isolated and installed cases are identical. At the bypass and core stream inlets, total pressure and total temperature conditions are imposed. At the far field, ambient conditions and a flight stream of $90 \mathrm{~ms}^{-1}$ is set. The nozzle diameter $D=0.228 \mathrm{~m}$. The isolated and installed nozzles are abbreviated as Iso. and Inst. respectively. The flight stream and flap deflection for each case are denoted FS and F followed by the flight stream velocity $(\mathrm{m} / \mathrm{s})$ and flap deflection angle (degrees) respectively. The coordinate system used is shown in Fig. 5 along with the azimuthal locations of the sideline (SL) and flyover (FO) profiles. 

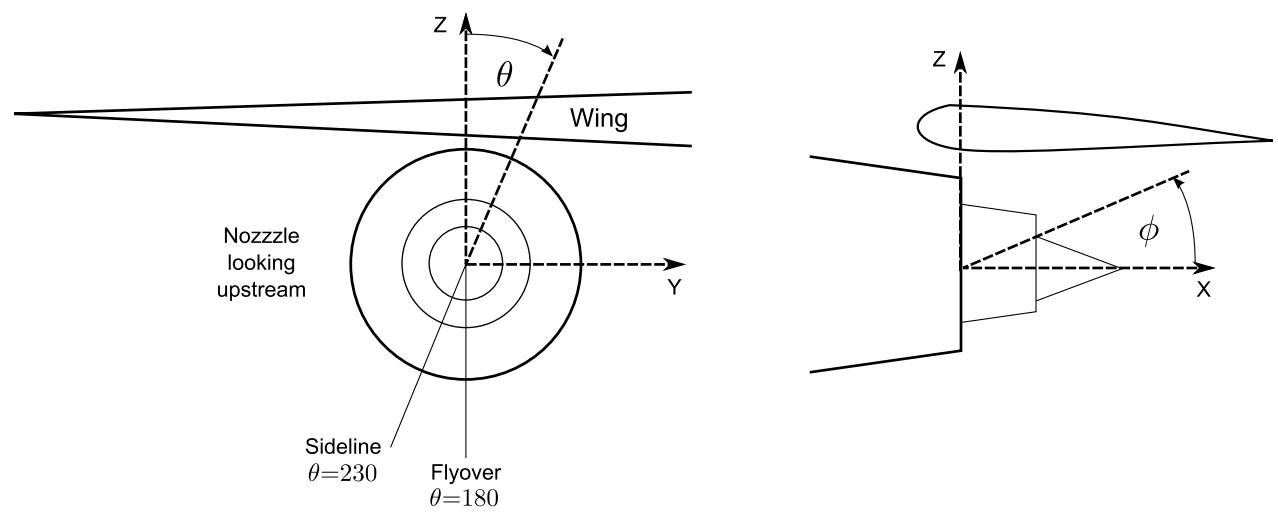

Figure 5. Coordinate system showing azimuthal (left) and polar (right) coordinates.

\section{Results}

\section{A. Instantaneous and Mean Flow}

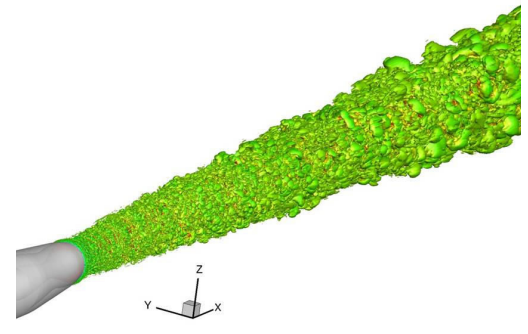

(a) Iso. FS90

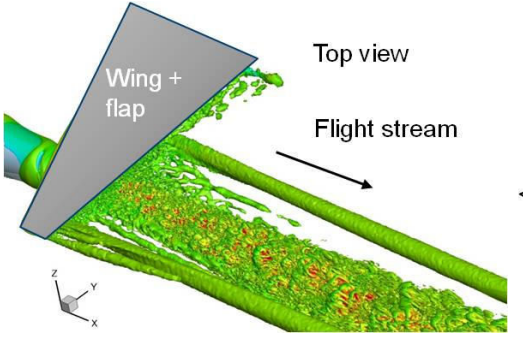

(b) Inst. FS90 F14 top

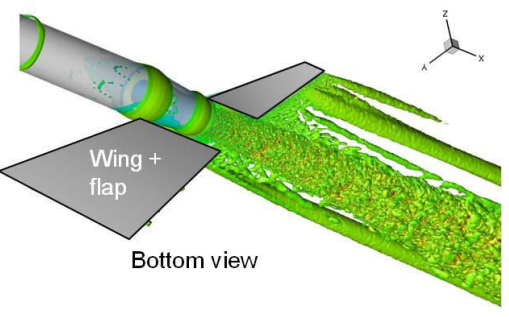

(c) Inst. FS90 F14 bottom

Figure 6. Q-criterion coloured by axial velocity for isolated (Iso.) and installed case with $14^{\circ}$ flap deflection.

Figure 6 shows Q-criterion isosurfaces for both isolated and installed cases. The installed cases generate a complex flow and the flight stream convects vorticity from the jet, flap and wing tip far downstream.

Figure 7 shows axial velocity contours in the $y=0$ plane for the cases studied. The installed nozzle with a $14^{\circ}$ flap deflection shows the jet plume initially sucked towards the wing then deflected downwards by around $0.5 D$ at $x / D=15$. For the flap deflection of $8^{\circ}$, the effect is minimal, indicating the case may be used to contrast the presence of surfaces near the nozzle generating new sources and reflections and more dramatic modification of the jet plume as with the higher flap deflection.

\section{B. Velocity and turbulence profiles}

Figure 8(a) displays the axial velocity development along the a streamline starting at the plug centre (equivalent to the isolated jet centreline). These are indicated in Fig. 7 green lines. For the isolated case, downstream of the plug, the velocity rises rapidly before a more gradual rise as density variations due to hot core flow and mixing of the bypass flow produce a peak at $x / D=5$, before a slow decay as a result of the flight stream. This is in contrast to single stream hot jets without flight stream, which begin with the maximum velocity and decay rapidly. The isolated profile is modified by the wing and flap. The downstream decay of the F8 case is similar to the isolated case. The F14 decay is faster, reaching $u / u_{c}=0.5$ around $5 D$ further upstream than the F8 case. As shown in Fig. 8(b), the axial Reynolds stresses of the F8 and F14 cases are increased by approximately $50-75 \%$ relative to the isolated case at $x / D=2.5$. This is the location where the inner and outer shear layers begin to merge and is also in the vicinity of the flap trailing edge for the installed cases $(x / D \approx 2.1$ at the flap centre). The second, broader peak, corresponds to the collapse of the potential core. The peak location and turbulence level for the isolated and F8 cases are similar and broader 


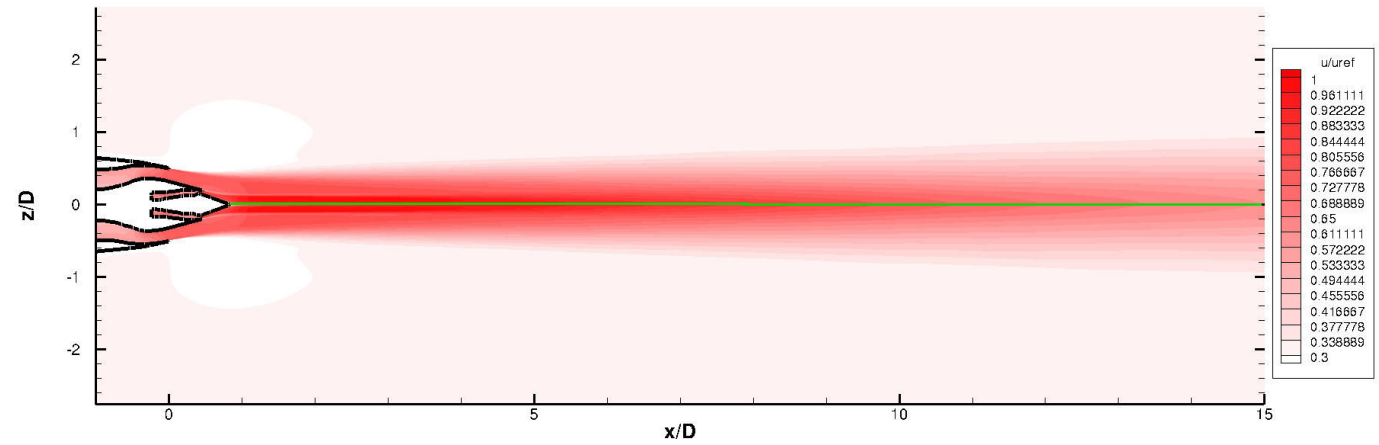

(a) Iso. FS90

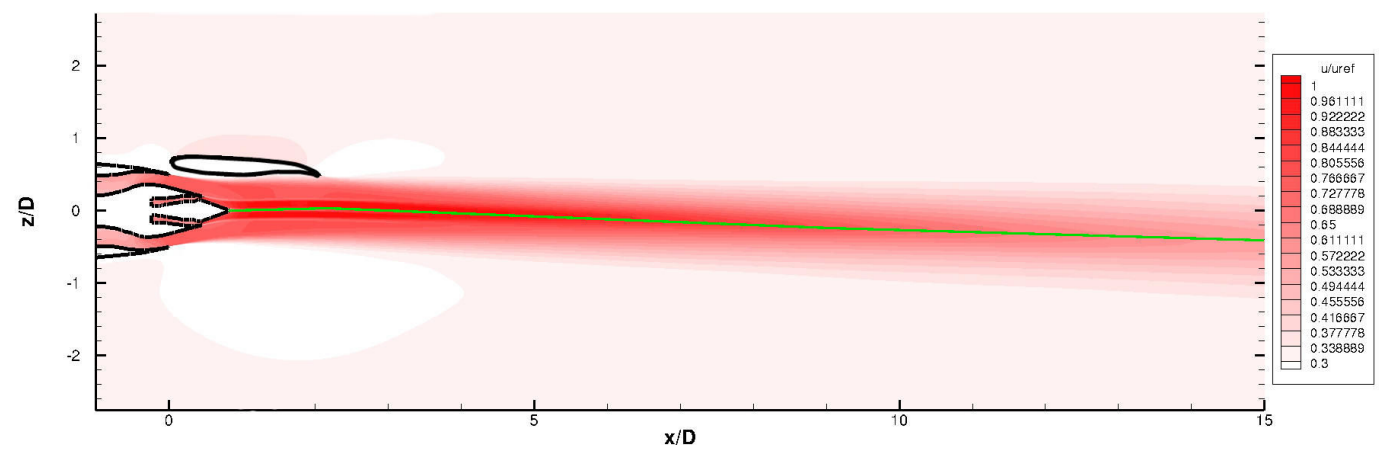

(b) Inst. FS90 F14

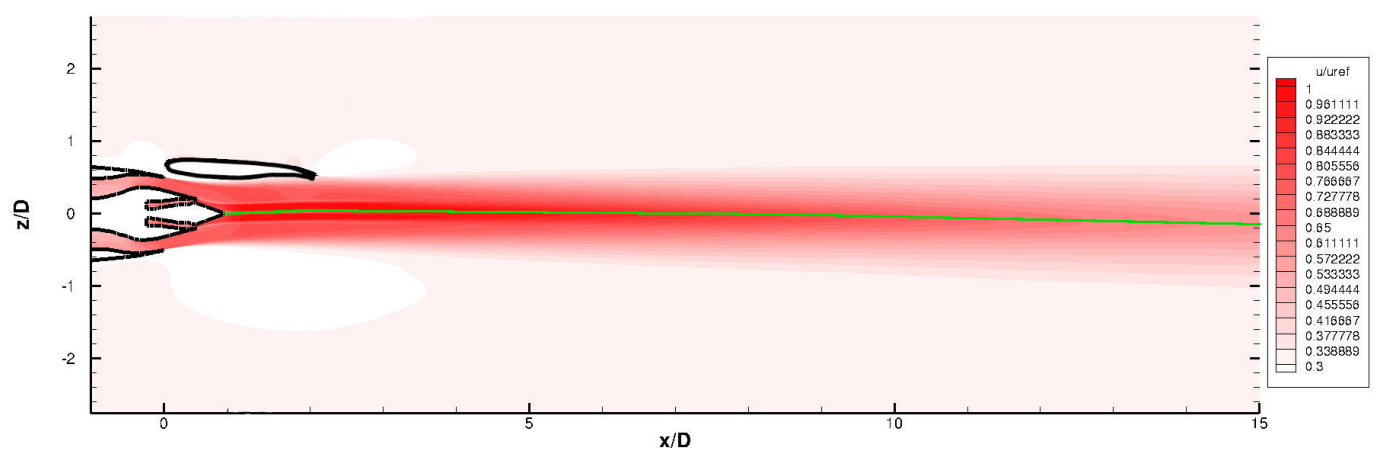

(c) Inst. FS90 F8

Figure 7. Axial velocity contours for the isolated and installed cases at the $y=0$-plane. Streamlines are indicated in green to provide an equivalent centreline profile. 


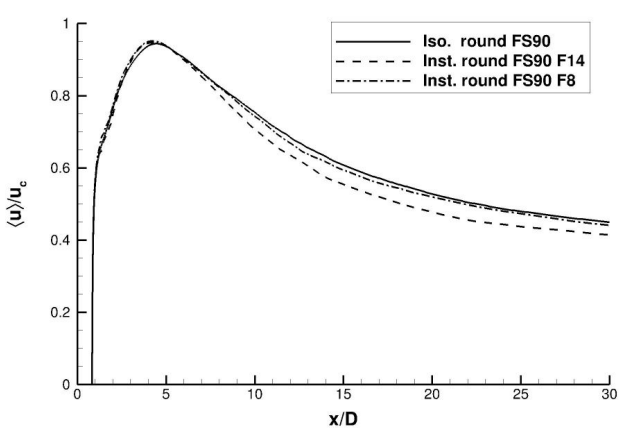

(a) Axial velocity

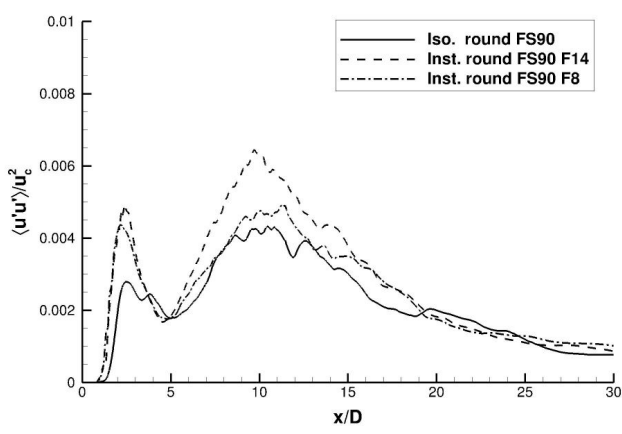

(b) Axial Reynolds stress

Figure 8. Axial velocity and Reynolds stress profiles along a streamline from the plug centre.

at $9<x / D<12$. The F14 case again increases the peak Reynolds stress by $50 \%$ and creates a sharper peak at $x / D=10$. After $x / D=15$, stress levels are similar for all cases.

Figure 9 shows axial Reynolds stress profiles at $y=0$ and $z=0$ planes. Figure 9 (a) shows that for installed cases, just downstream of the flap trailing edge $(x / D \approx 2.1)$, the stress is nearly doubled relative to the isolated nozzle. The higher flap deflection broadens the increased turbulence level into the main jet plume as far as $7.5 \mathrm{D}$ then merges with the jet plume increasing overall turbulence levels. The lower flap deflection shows a small increase at $z / D \approx 0.25-0.5$ until $x / D=5$ but becomes mixed into the main jet plume and is then similar to the isolated case. In the $z=0$ plane below the wing (Fig. 9(b)), the F14 case shows an increase and broadening of the Reynolds stress profile. Note the installed profiles are not symmetrical due to the finite span tapered wing.

Similar trends for the shear stresses are displayed in Fig. 10. The peak in the shear stress in Fig. 10(a) is at a lower $z / D$ position than the isolated of $\mathrm{F} 8$ cases due to greater flow deflection. An increase in shear stress is clearly visible at $8-10 D$ where the potential core collapses. Fig. 10(b) shows little effect from the wing and flap except a small increase and profile broadening over the isolated case.

\section{Space-time correlations}

Second and fourth order space-time correlations are extracted from unsteady data to reveal changes in length and time scales and dominant sources. The formulae are as follows

$$
\begin{gathered}
R_{i j}(x, \Delta, \tau)=\frac{\left\langle u_{i}^{\prime}(x, t) u_{j}^{\prime}(x+\Delta, t+\tau)\right\rangle}{\left\langle u_{i}^{\prime}(x, t) u_{j}^{\prime}(x, t)\right\rangle} \\
R_{i j k l}(x, \Delta, \tau)=\frac{\left\langle\left(\rho u_{i}^{\prime} u_{j}^{\prime}-\left\langle\rho u_{i}^{\prime} u_{j}^{\prime}\right\rangle\right)(x, t) \cdot\left(\rho u_{k}^{\prime} u_{l}^{\prime}-\left\langle\rho u_{k}^{\prime} u_{l}^{\prime}\right\rangle\right)(x+\Delta, t+\tau)\right\rangle}{\left\langle\left(\rho u_{i}^{\prime} u_{j}^{\prime}-\left\langle\rho u_{i}^{\prime} u_{j}^{\prime}\right\rangle\right)(x, t) \cdot\left(\rho u_{k}^{\prime} u_{l}^{\prime}-\left\langle\rho u_{k}^{\prime} u_{l}^{\prime}\right\rangle\right)(x, t)\right\rangle}
\end{gathered}
$$

Second order space-time correlations in the outer shear layer (at $\theta=0$ ) are provided in Fig. 11 at different axial locations. The gradient of the contours shown indicate the convective speed of vortices in the flow. This varies from approximately $0.6 u_{c}$ at $x / D=3$ to $0.5 u_{c}$ at $x / D=20$. There appears to be no significant change in length or time scales between the isolated and F14 cases.

Figure 12 shows $4^{t h}$-order space time amplitudes for the three cases studied at $x / D=3$ downstream of the flap for installed cases. These represent the magnitude of Goldstein analogy sources and can also be used to improve acoustics models. ${ }^{20}$ For each significant component an increase in magnitude is evident when the wing-flap is included with. This is most significant with the F14 case. The installed cases show over $100 \%$ increase in R1111 compared to the isolated jet. For a more detailed picture other locations will need to be assessed. 


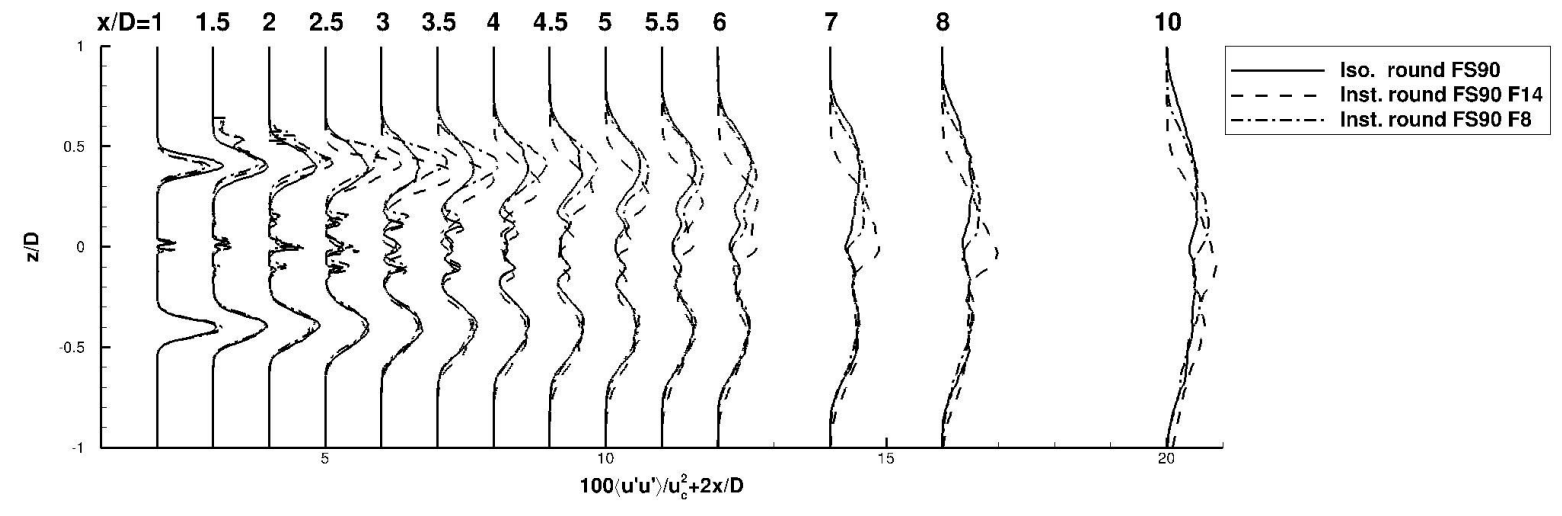

(a) $y=0$ plane

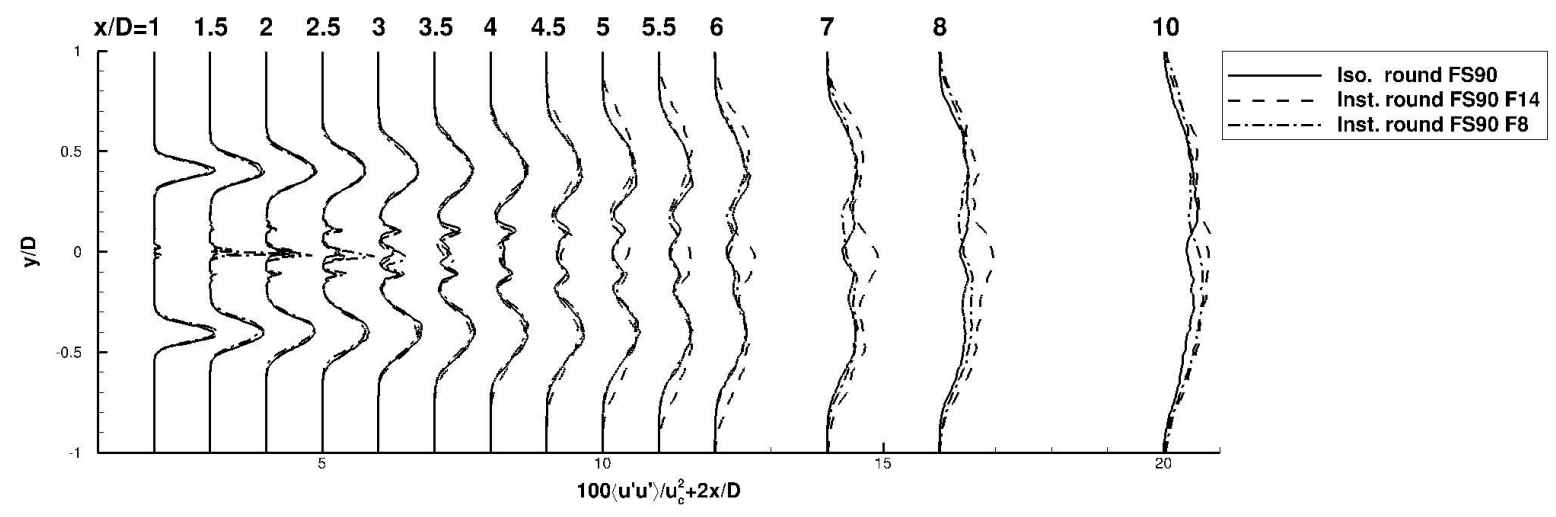

(b) $z=0$ plane

Figure 9. Reynolds axial stress profiles at $y=0$ and $z=0$ planes. 


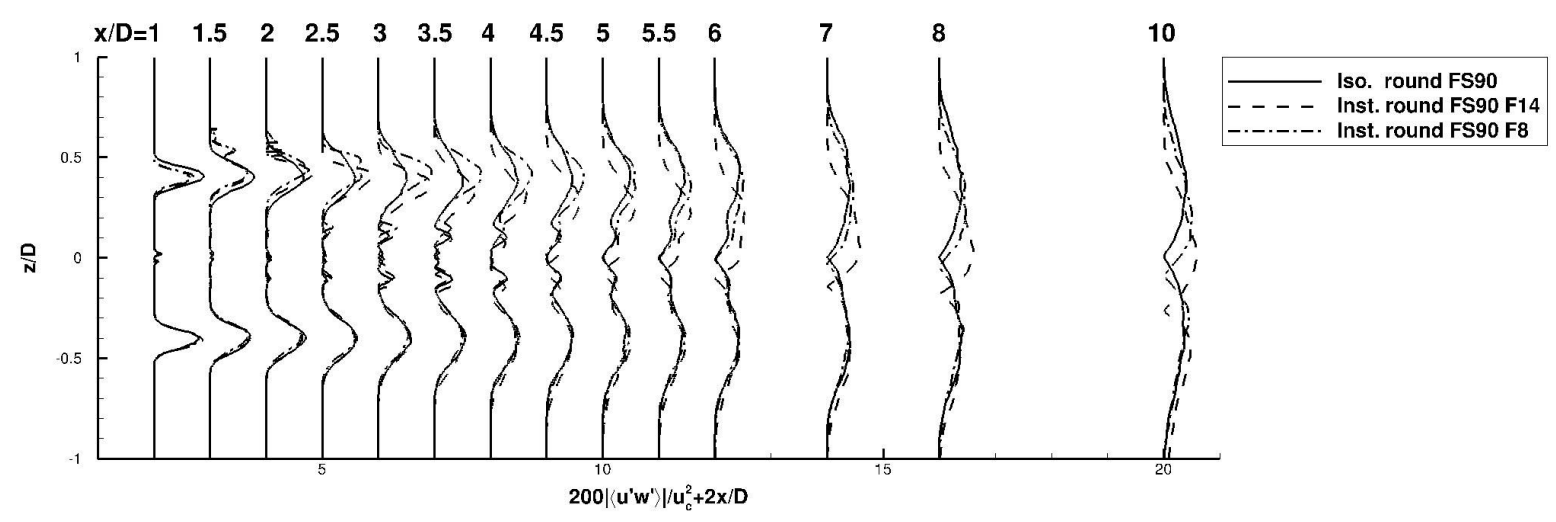

(a) $y=0$ plane

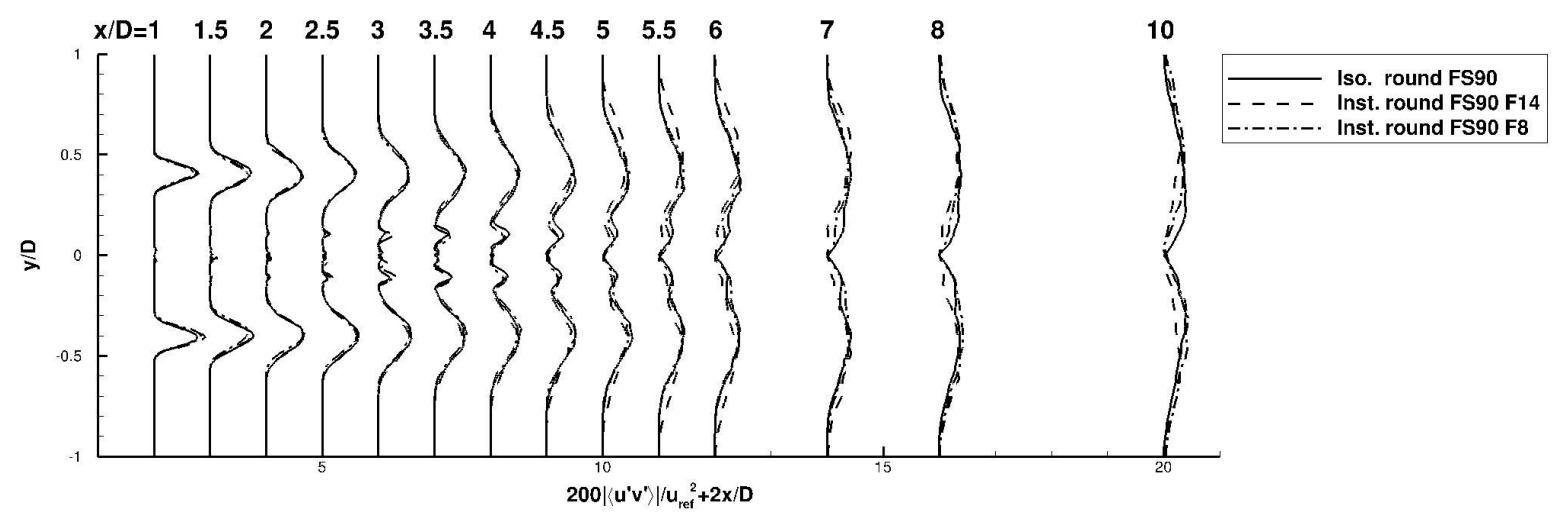

(b) $z=0$ plane

Figure 10. Reynolds shear stress profiles at $y=0$ and $z=0$ planes.

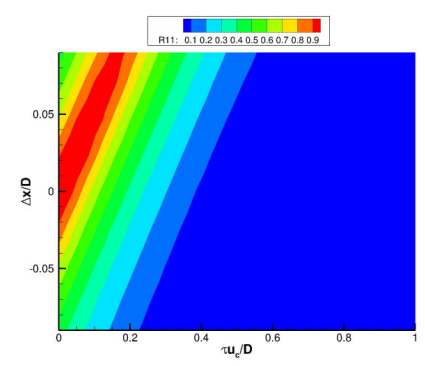

(a) Iso. $x / D=3$

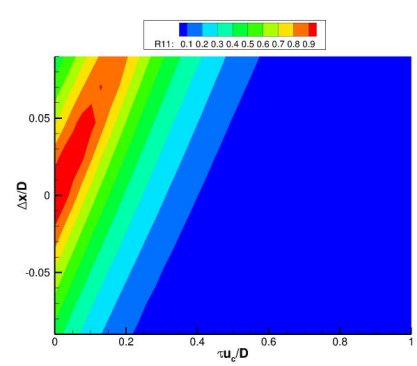

(d) $\mathrm{F} 14 x / D=3$

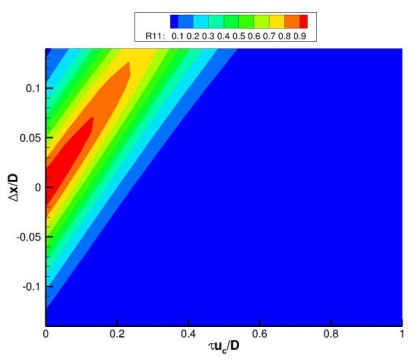

(b) Iso. $x / D=10$

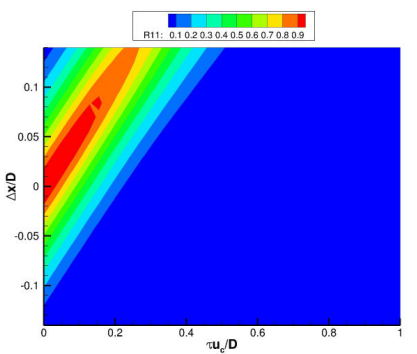

(e) F14 $x / D=10$

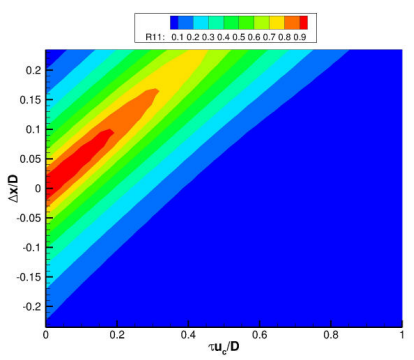

(c) Iso. $x / D=20$

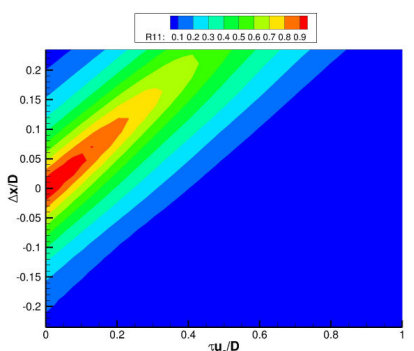

(f) $\mathrm{F} 14 x / D=20$

Figure 11. Second order space-time correlation contours along the outer shear layer. 


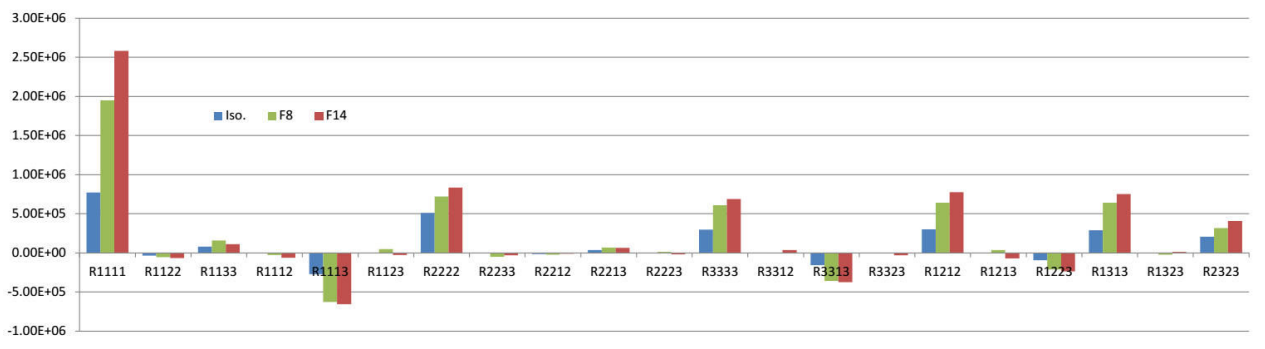

Figure 12. $4^{\text {th }}$-order space-time correlation componenets at $x / D=3$ in the outer shear layer at $\theta=0$.

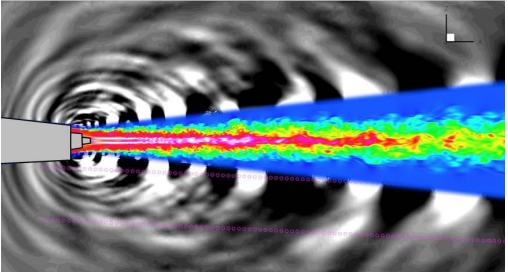

(a) Iso. FS90 $y=0$ plane

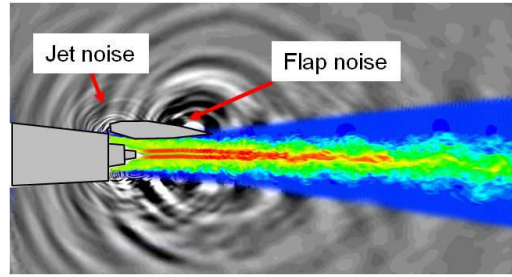

(b) Inst. FS90 F14 $y=0$ plane

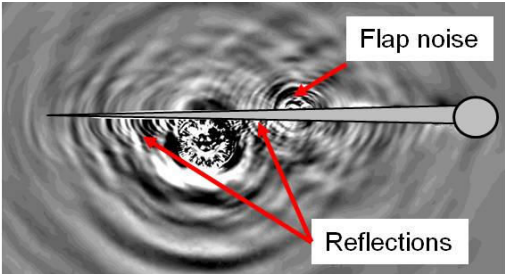

(c) Inst. FS90 F14 $x \approx 1 D$ plane

Figure 13. Instantaneous $d p / d t$ contours for the isolated and F14 case at different plane locations.

\section{Acoustics}

Figure 13 displays $d p / d t$ contours for the isolated and installed nozzle (F14). The isolated nozzle (Fig. 13(a)) shows a fairly spherical source with waves convected downstream. For the installed case in Fig. 13(b), the flap clearly generates a large local noise source. This region is also known to refract acoustic waves over the wing. The location of the installed nozzle allows high frequency waves to reflect off the wing leading edge. Figure 13(c) clearly shows reflection of waves off the underside of the wing and small and large scale acoustic waves emanating from the flap region. Of interest is the effect of turbulence ingested from the jet shear layer into the flap recess, where the flow is then accelerated before exiting along the flap upper surface. This is akin to slat noise as shown by Deck and Laraufie. ${ }^{21}$

Farfield sound is calculated at $12 \mathrm{~m}$ using the FWH method and back propagated to a distance of $1 \mathrm{~m}$ using emission coordinates.

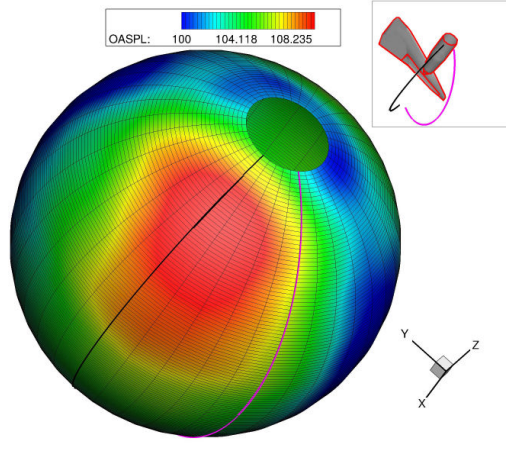

(a) F14

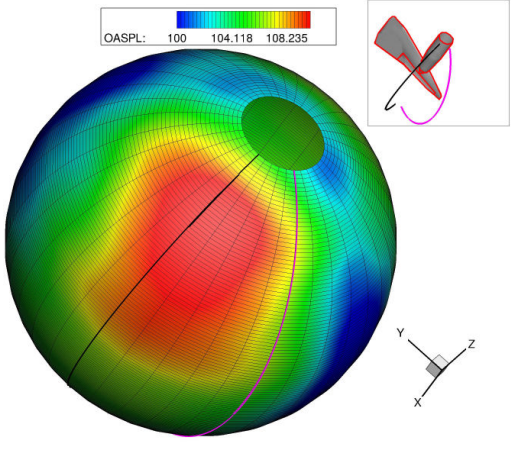

(b) F8

Figure 14. Farfield OASPL in $\phi$ (polar) and $\theta$ (azimuthal) angles projected onto a sphere surrounding the jet. The orientation is indicated by the inset geometry figure and axes. Flyover and sideline profiles are indicated with black and magenta lines respectively.

Figure 14 shows the farfield OASPL around the F14 and F08 cases beneath the wing. The OASPL is projected onto a sphere using polar and azimuthal coordinates. The key flyover and sideline profiles are 
indicated. The wing-flap produces complex jet-surface interaction. The flap trailing edge is a significant noise source absent from the isolated case. At the wing inboard and outboard directions, sound waves are blocked by the wing-flap geometry and noise is instead reflected downwards. Most noise is hence directed beneath the wing as shown in Fig. 14. Above the wing (not shown), installation effects create more upstream noise but this region does not receive reflected noise from the jet plume. Most installation noise appears in the upstream direction $\left(\phi>90^{\circ}\right)$. The flyover profile passes near the maximum OASPL for both installed cases. The sideline profile lies just outside the noisiest region. The main difference between the two flap deflections appears to be a small change in directivity.

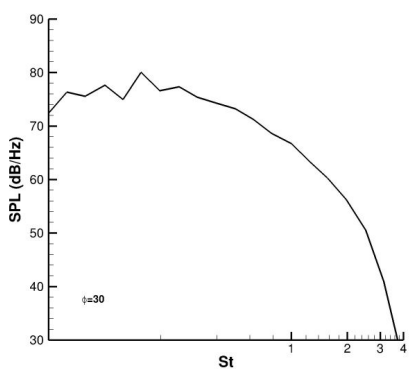

(a) Iso. $\phi=30$

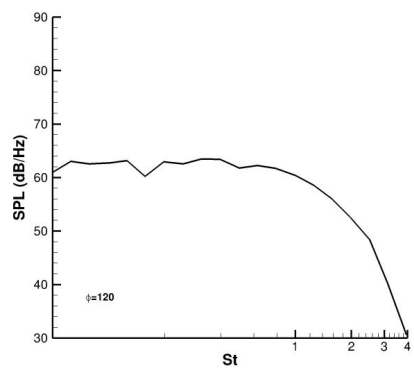

(d) Iso. $\phi=120$

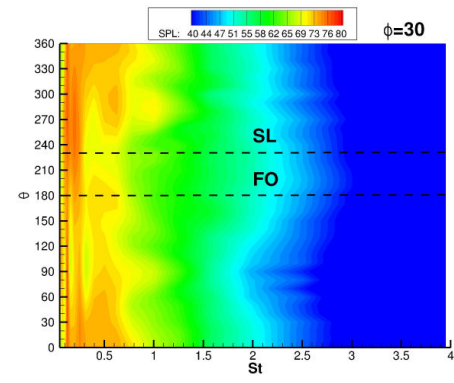

(b) $\mathrm{F} 8 \phi=30$

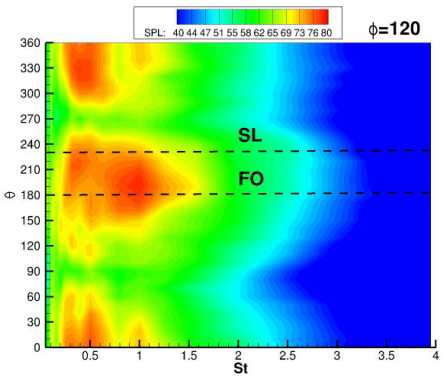

(e) $\mathrm{F} 8 \phi=120$

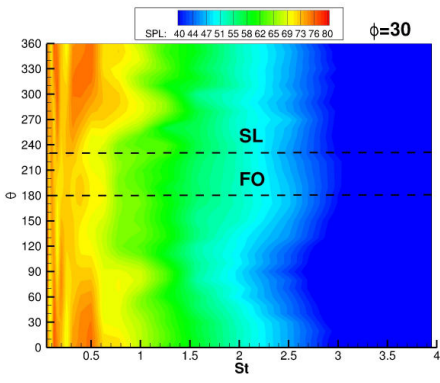

(c) $\mathrm{F} 14 \phi=30$

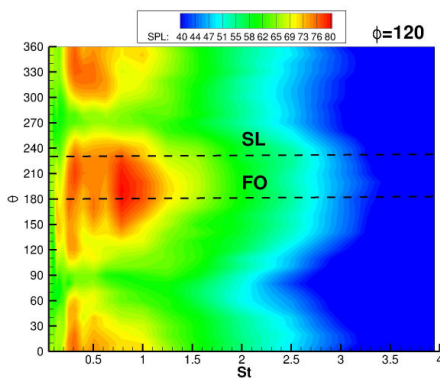

(f) $\mathrm{F} 14 \phi=120$

Figure 15. SPL contours plotted against azimuthal position and St.

The spectral content of the noise produced is shown in Fig. 15. The isolated case is azimuthally averaged. The variation of SPL contours with azimuthal location and Strouhal number $\left(S t=f D / u_{c}\right)$ for installed cases at two different polar angles is presented. At $\phi=30^{\circ}$ (Fig. 15(a)-15(b)), there is relatively low influence from the upstream wing-flap geometry except for two weak peaks and most noise is in the low frequencies $(S t<1)$. The peak SPL is similar to the isolated case. At $S t=0.5$ the F14 case shows a higher SPL than the F8 case. At $\phi=120^{\circ}$ (Fig. 15(d)-15(f)) the azimuthal variation becomes more defined and both high and particularly low frequencies are more prominent below $(\theta=180)$ and above $(\theta=0)$ the wing. This shows a strong upstream influence due to installation effects.

\section{Conclusion}

Flow and noise produced by a round, ultra-high bypass-ratio aeroengine nozzle with a hot core and outer flight stream has been studied. Additionally, installation effects for a wing-flap geometry with two flap deflection angles have been investigated. LES-RANS provides detailed data for modern engine nozzles and jet-airframe interaction. Such detailed data is difficult to acquire experimentally. Installation effects introduce significant noise directionality in the azimuthal direction and alters the spectral composition, particularly at upstream polar angles. Second order space-time correlations provide spatial and temporal scales. Using fourth order space-time correlations, changes in source strength and location can be identified. These can be used to improve acoustics models. It is expected that further analysis will help unlock noise generation mechanisms to aid design. 


\section{Acknowledgments}

The authors would like to acknowledge the collaboration with C. Mockett of CFD Software E-F GmbH, Berlin, for the hybrid meshing strategies presented. Computing time is greatly appreciated from both the United Kingdom Turbulence Consortium (UKTC), EPSRC EP/L000261/1 and the UK national computing facility ARCHER. The work was performed in the EU-funded project JERONIMO (ACP2-GA-2012-314692JERONIMO).

\section{References}

${ }^{1}$ Viswanathan, K., Czech, M. J., and Lee, I. C., "Towards Prediction of Dual-Stream Jet Noise: Database Generation," 49th AIAA Aerospace Sciences Meeting including the New Horizons Forum and Aerospace Exposition, No. January, AIAA, Orlando, Florida, 2011.

${ }^{2}$ Mendez, S., Shoeybi, M., Lele, S. K., and Moin, P., "On the use of the Ffowcs Williams-Hawkings equation to predict far-field jet noise from large-eddy simulations," International Journal of Aeroacoustics, Vol. 12, No. 1+2, 2013, pp. 1-20

${ }^{3}$ Wang, Z.-N., Naqavi, I. Z., Mahak, M., Tucker, P., Yuan, X., and Strange, P., "Far Field Noise Prediction of Subsonic Hot and Cold Jets Using Large-Eddy Simulation," Proceedings of ASME Turbo Expo 2014, ASME, Dusseldorf, Germany, 2014, pp. $1-11$.

${ }^{4}$ Nelson, C., Cain, A., Du, Y., Morris, P., and Spyropoulos, J., "Toward Efficient Computational Aeroacoustic Analysis of High Speed Jets," ASME Turbo Expo 2013, ASME, San Antonio, Texas, 2013, pp. 1-14.

${ }^{5}$ Tyacke, J. C., Naqavi, I. Z., Wang, Z.-N., Tucker, P. G., and Boehning, P., "Predictive Large Eddy Simulation for Jet Aeroacoustics - Current Approach and Industrial Application," Journal of Turbomachinery, Vol. 139, No. 8, 2017, pp. 081003081003-13.

${ }^{6}$ Paliath, U. and Premasuthan, S., "Large Eddy Simulation for Jet Installation Effects," 19th AIAA/CEAS Aeroacoustics Conference, may 2013, pp. 1-10.

${ }^{7}$ Langtry, R. B., Larssen, J. V., Winkler, C. M., Dorgan, a. J., and Mani, M., "DDES and Acoustic Prediction of Rudimentary Landing Gear Experiment Using Unstructured Finite Volume Methods," Flow, Turbulence and Combustion, Vol. 91, No. 3, sep 2013, pp. 717-745.

${ }^{8}$ Rahier, G., Huet, M., and Prieur, J., "Additional terms for the use of Ffowcs Williams and Hawkings surface integrals in turbulent flows," Computers and Fluids, Vol. 120, 2015, pp. 158-172

${ }^{9}$ Pérez Arroyo, C., Daviller, G., Puigt, G., and Airiau, C., "Hydrodynamic - Acoustic Filtering of a Supersonic Underexpanded Jet," ERCOFTAC workshop Direct and Large- Eddy Simulation 10, 2015, pp. 1-6.

${ }^{10}$ Jameson, A., "Formulation of Kinetic Energy Preserving Conservative Schemes for Gas Dynamics and Direct Numerical Simulation of One-Dimensional Viscous Compressible Flow in a Shock Tube Using Entropy and Kinetic Energy Preserving Schemes," Journal of Scientific Computing, Vol. 34, No. 2, dec 2007, pp. 188-208.

${ }^{11}$ Tyacke, J. C., Mahak, M., and Tucker, P. G., "Large-Scale Multifidelity, Multiphysics, Hybrid Reynolds-Averaged NavierStokes/Large-Eddy Simulation of an Installed Aeroengine," Journal of Propulsion and Power, Vol. 32, No. 4, 2016, pp. $997-1008$

${ }^{12}$ Naqavi, I. Z., Wang, Z.-N., and Tucker, P. G., "Far-field noise prediction for jets using Large-eddy simulation ( LES ) and Ffowcs Williams-Hawkings ( FW-H ) method," International Journal of Aeroacoustics, Vol. 15, No. 8, 2016, pp. 1-29.

${ }^{13}$ Eastwood, S., Tucker, P., Xia, H., and Klostermeier, C., "Developing Large Eddy Simulation for Turbomachinery Applications," Phil. Trans. R. Soc. A, Vol. 367, No. 1899, 2009, pp. 2999-3013

${ }^{14}$ Eastwood, S., Hybrid LES RANS of Complex Geometry Jets, Phd thesis, University of Cambridge, 2009

${ }^{15}$ Spalart, P. R. and Allmaras, S. R., "A one-equation turbulence model for aerodynamic flows," La Recherche Aérospatiale, Vol. 1, No. 1, 1994, pp. 5-21.

${ }^{16}$ Liu, Y., Tucker, P. G., and Kerr, R. M., "Linear and nonlinear model large-eddy simulations of a plane jet," Computers and Fluids, Vol. 37, 2008, pp. 439-449.

${ }^{17}$ Smagorinsky, J., "General circulation experiments with the primitive equations. I. The basic experiment," Monthly Weather Review, Vol. 91, No. 3, 1963, pp. 99-165.

${ }^{18}$ Najafi-Yadzi, A., Bres, G. A., and Mongeau, L., "An acoustic analogy formulation for moving sources in uniformly moving media," Proc. R. Soc. A, 2010, pp. 1-22.

${ }^{19}$ Mockett, C., Fuchs, M., Kramer, F., Michel, U., and Thiele, F., "Turbulence Modelling and Meshing Developments for the Prediction of Jet Noise Installation Effects," 22nd AIAA/CEAS Aeroacoustics Conference, AIAA/CEAS, Lyon, 2016, p. 2933.

${ }^{20}$ Karabasov, S. A., Afsar, M. Z., Hynes, T. P., Dowling, A. P., McMullan, W. A., Pokora, C. D., Page, G. J., and McGuirk, J. J., "Jet Noise: Acoustic Analogy Informed by Large Eddy Simulation," AIAA Journal, Vol. 48, No. 7, 2010, pp. 1312-1325.

${ }^{21}$ Deck, S. and Laraufie, R., "Numerical investigation of the flow dynamics past a three-element aerofoil," J. Fluid Mech., Vol. 732, 2013, pp. 401-444. 